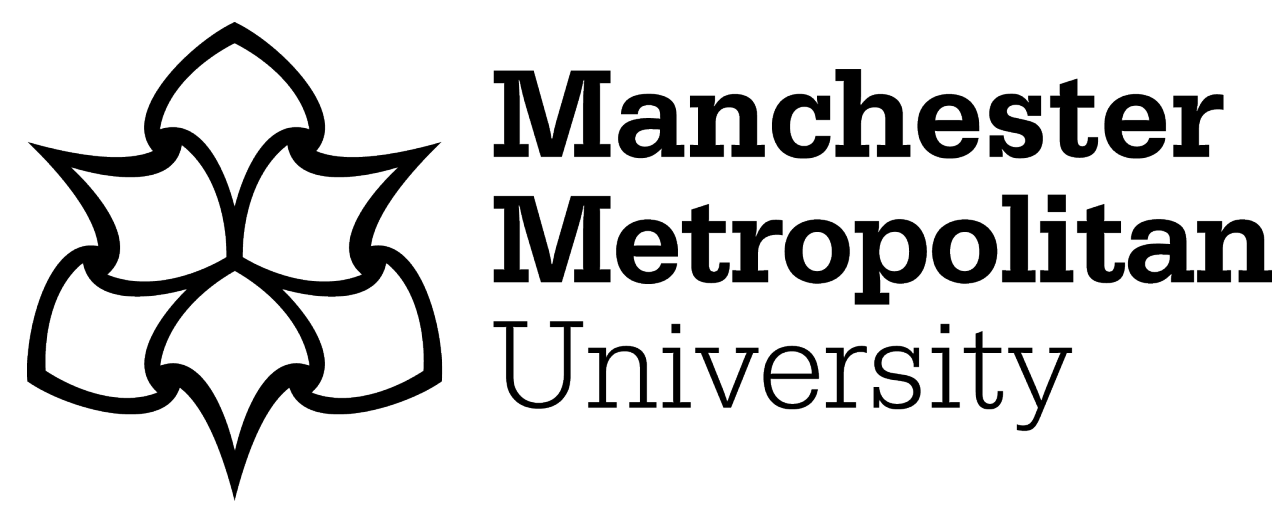

Brown, Tony (1994) Creating evidence towards making statements about classroom activity. Research in Education, 51 (1). pp. 33-40. ISSN 00345237

Downloaded from: https://e-space.mmu.ac.uk/622349/

Version: Accepted Version

Publisher: SAGE Publications

DOI: https://doi.org/10.1177/003452379405100104

Please cite the published version 


\section{CREATING EVIDENCE TOWARDS MAKING STATEMENTS ABOUT CLASSROOM ACTIVITY}

\section{TONY BROWN}

\section{INTRODUCTION}

In this paper I examine some of the issues underlying the creation of data in classroom based research. In particular, I focus on the status of evidence offered by a researcher describing children's activity in order to demonstrate how the researcher's interest necessarily conditions the various stages of any enquiry. I show how evidence is created through a process of selection and interpretation consequential to the researcher making assumptions about the situation he or she observes. I also show how the descriptions themselves, as offered by the researcher, become a material part of the situation being described. In the first section I consider the issues arising in the identifying and gaining access to lessons. This is followed by a discussion about the generation of data. Finally, I examine some of the difficulties to be faced in interpreting such data.

\section{GAINING ACCESS TO LESSONS}

In setting up a classroom based research enquiry the selection of any particular lesson is in itself clearly problematic. It seems completely inadequate to speak of representative lessons in representative schools. My purpose in describing individual schools is to capture features which I hope others will recognise and which will in some way resonate with situations they have met in their own work. In setting out on a research enquiry I might have some specific problem in mind, but in framing the problem I have necessarily made some assumptions about the structure of the situation. Any question is rooted in my current knowing and described in the verbal categories I presently employ in such situations. In gaining a new perspective on learning situations it seems necessary that I acknowledge a possible instability in the linguistic categories I employ in my descriptions. In identifying a lesson for my enquiry I am attending to certain features that I currently describe in a particular way. However, any work in respect of this lesson brings in to question this mode of describing. These changes in myself clearly affect any statements I make subsequently in to a wider community. I do not, however, see changes in myself stopping here.

A few years ago, I carried out an extensive programme of classroom based fieldwork, together with some teachers, in the Commonwealth of Dominica (described in Brown, 1987). The changes I underwent myself, from being a new arrival towards feeling able to make statements about specific lessons relating to my research enquiry, seemed substantial. Initially I had to deal with jet lag, culture shock and racism. Getting to the schools often involved walking for hours through rain-forests and sitting in wet clothes. I was accommodated in homes which were very welcoming but also very different. I witnessed lessons where there were no facilities other than broken furniture. I had to 
witness corporal punishment. I had not worked with teachers as a college tutor before. People could not understand my London accent when I spoke to them. It may be suggested that this is not a typical research project but any greater comfort I might feel in tackling lessons in familiar UK locations can only be explained by greater familiarity. Such familiarity, however, can only highlight the number of assumptions we normally make about situations we observe and in research questions we pose in respect of them. Pool (1989) discusses the difficulty of using the verbal categories of one culture to describe activity in another. In his own project where, as a teacher from an English background, he faced working in Sierra Leone, he suggested that the verbal categories of the two cultures are incommensurate and that moving from one to the other requires a lengthy initiation.

Changes in lessons observed also seem inevitable. During the Dominica project I declared myself either as a college tutor or simply as an observer. The teachers accomodated me to some extent by making subtle changes to their lessons and the children's behaviour was perhaps slightly different since they knew I was watching them. It seemed pointless for me to claim invisibility since I could only come to know the lesson through being an involved human participant, making various assumptions about what was happening both in advance and along the way. Even if it were possible for a researcher not to disturb a lesson with his or her presence it is necessarily disturbed in any subsequent interpretations made.

\section{GENERATING DATA}

As someone observing a lesson, I am investigating phenomena that transcend the signifiers in my immediate perception; I can only be aware of a very small part of that which takes place. There is a temptation to find approaches that help me to record the largest possible amount of data. There are, however, considerable risks in collecting too much data since in being exposed to multiple perspectives I may find it impossible to reconcile them. The interpretation of the data may well depend on the order in which it is tackled; the context for interpretation of each element depending on what has been seen already. For example, if I re-read a certain section of transcript my anticipations and recollections with respect to elements I perceive to be in it will be quite different to those I had in my initial reading. Interpretation then needs to be held in suspension as it is not possible to interpret a word or a sentence in the moment, as the context of that word or sentence does not reveal itself simultaneously. Such a context will continuously change as reading proceeds, and further as one reflects on it, and as it is assimilated with one's experience.

In writing lesson reports I can only record a small part of that which I attend to and as I record I cannot know the significance that I will later place on each element I identify. This will be true of any approach to recording and in particular challenges the notion of following a method. A method of recording, such as an observation schedule (e.g. Beeby et al 1979) necessarily presupposes a form for that being watched which results in all

observations being seen and recorded according to a preconceived scheme. This further 
presupposes a way of watching associated with certain notions of 'teachers', 'lessons' and 'children' etc, emphasising those features seen as significant by the schedule designers. With such an approach, many interpretations arise in the journey from the 'actual' lesson, through the researcher filling in the schedule to the various stories that might be told in respect of the completed schedule.

Video techniques, which have been discussed more fully elsewhere (e.g. Brown 1990), have been used widely by many researchers but this technique of recording seems no less fraught with potential problems. There is a substantial change in the transition from being an observer present at the lesson to being a viewer of the video recording. Such a recording is made by a camera operator within the technical possibilities and it is unable to replicate the visual perspective of any participant. The operator is also unable to predict the structure she or any other viewer will subsequently assign to the lesson in their viewing of the film.

\section{INTERPRETING DATA}

In coming to know about a particular lesson I may come away from it with memories of my involvement and perhaps some tangible product such as videotape or manuscripts which might serve as aids to memory. My task is to decide how I might combine elements of my memory towards making statements. It may be that my research question has become modified in the enquiry so far and may be subject to further change as the research product is scrutinised. This product comprises memories that are now seen in the context of each other and some of these may fade as new structures placed on them marginalise certain features. It also may comprise some tangible 'record'. I assess this product in the context of my experiences and in the light of my current motives.

I shall set out by exploring the nature of the reduction that takes place in attempting to record the 'complete' lesson in the form of a transcript and then go on to consider what might be called evidence. I include here a brief extract of a transcript created during a lesson given by one of the teachers involved in the Dominica project. The account was recorded by the teacher and is re-produced here in its original form.

Four six year olds sit around a table together. All speech recorded.

Teacher: Take your exercise books and pencils. Work together using the counters and make as many stories of 12 as you can. Write the number stories.

Richardson: 1,2,3,4,5,6,7,8,9,10,11,12! (as he touches each counter). Clifford : 3 plus $1,2,3,4,5,6,7,8,9,10$ ! (as he touches) 
Richardson: (to Clifford) 11,12.

(to himself) 3 plus $1,2,3,4,5,6,7,8,9$ (as he points)

Roger : Nine plus what? (He then writes $9+3=12$ since he was ignored)

Chester : How 12 going?

Roger : A one and a two.

Chester : 10 plus 2 is 12 (He then writes it in his book)

Clifford : 10 plus 2

Chester : and 2 plus 10

Richardson: 2 plus 10? Boy, you do matching (they then write $2+10=12$ )

Clifford : 1 plus (as he manipulates bottle tops he looks to Richardson)

Richardson: 1,2,3,4,5,6,7,8,9,10,11. 1 and 11. (They all write it)

Clifford : Look it 11, a 1 and a 1.

Chester : 12 plus 1 ?

Roger : 12 plus 1? Whey! (sarcastically)

$: 11$ plus 1.11 plus 1 is 12 .

The few sentences of the report to some extent evoke the lesson which 'actually' occurred. Some elements of the lesson perceived have been transformed reductively into the elements of the lesson report. In reading the report we invert this transformation so that we can imagine the actual lesson. The nature of this inversion, however, will depend on who the reader is. The teacher, one of the boys, various complete outsiders or I, would all have different ways of fulfilling the reference of the text in the report. The transformation that takes the actual lesson into the teacher's report is many-to-one whilst the inversion is one-to-many. The teacher, as the writer of the report, determines the rules of the original transformation by selecting the elements of the lesson that she records and how. The reader of her report chooses the rules by which the inversion is made according to their own scheme of interpretation. This interpretation will depend on the history and motives of the reader, along with the expectations associated with these. This demands further unfolding.

Ricoeur (1981), in his classic work on hermeneutics, sees text as a "fixation" of a speech event whereby elements of the event are reduced into a set of written words. He explores this transformation to consider what is lost from the 'actual' event and in so doing identifies various traits that differentiate the speech-event from its fixation in writing. I shall adapt my interpretation of these four traits to the example of this lesson in identifying that which is lost by reading the report rather than attending the lesson.

\section{a) The fixation of the discourse}

A transformation is made from the discourse (spoken and written) to the report. The writing fixes the discourse which in the lesson was a fleeting event, but what exactly is fixed? In the report the 'said' of the speech event has been recorded and also that written by the teacher and children. There might be a one-to-one mapping from the 'said' of the discourse to the report. However, the discourse exists in a context that is not so readily transformed in this way. 
b) Face to face observation of the speaker by the interlocutor

It is possible to identify some of that lost in this reduction. In the lesson we would be conscious of who is speaking. For example, the gestures of the boys have only their spoken element represented in the report. Intonations, delays, facial expressions and pointing fingers are only sometimes implied. The possibility of interpreting such gestures is lost.

Knowing the boys myself and their teacher I can speculate on further elements missing in the reduction, such as the comedy of the whole situation, the way in which the three boys regard each other, the earnestness of their commitment to the task. In short, the individuality of those present is largely lost. Such features would contribute to my personal interpretation but they are not identified in the report.

\section{c) Speaker and interlocutor in a shared world}

Furthermore, the speech refers to a world and the reference of the speech reported is represented in a reduced state. Much of the speech seems to refer to the bottle tops and the counting of them. By not being present we are deprived of the full context of the speech and of the context of that to which it refers. Consequently, in considering the lesson from the outside, we fulfil the reference of the recorded speech from our own experience. The references within the 'closed' world of the boys and the teacher are not shared. Also, the time structure and pacing is lost. Our only time markers are inherent in the sequencing of the sentences of the report. The nature of the spacing between the words, however, is not clearly represented.

\section{d) Accommodation of interlocutor by the speaker}

The speech is also addressed to someone. As someone writing notes in lessons myself I was conscious of causing some degree of disturbance by my presence. On occasions I caused shyness among the children, sometimes they asked me questions about my work or their work, sometimes I became the topic of conversation. In certain situations I spoke to the children, perhaps to relax them or to enquire about their work. Similarly, because I was a teacher trainer or a researcher, the teacher sometimes felt uneasy about me being there which sometimes led to the teacher behaving differently. In a sense the speech and behaviour of the teacher and children were acknowledging my presence. I was being accommodated as a person in attendance. Such accommodation would occur in some form for anyone in attendance but clearly not for someone reading a report of the lesson. That the interlocutor disturbs the lesson in such a way is inevitable. Further, the interlocutor may be more able to come to terms with a situation that responds to his or her actions. In short, it is not possible for the teacher or children to influence the opinion of someone reading the report of the lesson. They do not address the reader in their actions.

The reliance on manuscripts may seem rather stark but I feel that by focussing on what they take away, the subjectivity underlying their usage as evidence is more clearly asserted. Whilst video techniques used in recording lessons, for example, might appear to take away less, the issue of how we identify certain elements and make statements 
remains. To what extent can I call upon 'hard' evidence in making statements about lessons? How might I combine these statements towards making generalisations about children's learning in lessons? Interpretation seems inevitable since I necessarily engage as a participating human with a individual background of experience, within a certain cultural perspective, and a current set of motives. I may take elements of 'hard' evidence but I place these in the context of my overall view. Conversely, the lesson may be forever subject to re-interpretation but at some point I may feel the need to accept this and make general statements about it all the same. It seems to me this circularity arises whenever there is an attempt to make statements in respect of classroom activity. There is always a tension between any situation and statements made in respect of it (see Brown 1991).

\section{CONCLUSIONS}

Walkerdine (1984) has suggested that in our attempts to describe we often find ourselves creating the very thing we wish to describe. By putting our observations into verbal categories we are creating a view that becomes in some way fixed. She offered the example of a categorisation used in describing aspects of the 'new classroom' in the sixties which, through being stated, could be seen as serving a regulatory function. In this paper I have shown how there is always a difficulty in developing a way of talking which evolves with the changes in the way of seeing. Reflective describing of evolving phenomena necessarily requires some temporary fixing of the verbal categories employed. Further, the statements made within this reflective process become part of the context to which subsequent statements refer.

I have highlighted how, in making statements about a lesson, the identification of my interest is of central importance. My specific interest will depend on the role I am performing. For example, as a college tutor reporting on a student's lessons, my interest might be to engage in a dialogue with the student towards enabling them to be able to reflect on their classroom practice. If however, I am watching an Open University video of a lesson, I am not so much concerned with describing what happened but rather, with bringing to it my own experience so that I might develop more rigorous ways of talking about my own practice. My current task in this paper of finding out about children working on mathematics in classrooms is partly motivated by a desire for gaining experience of observing children so that I feel more able to share the sense of observations made by others in their own classrooms. This may, for example, be of value in working with student teachers as I explore with them their way of describing their practice, but also I may, when working with colleagues or in writing, intend to make more general statements that go beyond their relevance to a specific lesson.

I have also shown how statements can only be made by the researcher after negotiatiating a position which facilitiates a certain perspective. In order to achieve this the researcher necessarily makes certain assumptions about what needs to be said and what needs to be looked at to make this possible. I select lessons according to my current motives and my 
predictions regarding what these lessons might reveal. As a researcher my eye can never be innocent.

The very essence of a research enquiry is to make some reduction of the situation being studied towards making statements that might help others modify their practice in a productive way. The partiality engendered here is an essential part of the stressing and ignoring process underlying any description. Such descriptions necessarily display certain ideological qualities insofar as actions are suggested by the selection of that described. As a researcher any statements I make are a product of my own lived experience during the research enquiry but this very experience has been largely constructed by myself as researcher towards specific ends. I have shown how this not only affects the lesson being observed but also myself as researcher and my capacity to make statements.

In working on a research problem, which may be modified as a context emerges in my eyes, my relation to the research situation and the verbal categories I use may be revised as the enquiry progresses. The descriptive language employed necessarily displays an instability as it becomes a material part of the phenomena it is attempting to describe. Additionally, as a researcher I come to know the situation by seeing how it responds to my actions, which results in a change in the 'actual' situation, in the perception I hold of it and in any statements I make subsequently. The time dimension of this necessarily hermeneutic process results in any account having some autobiographical dimension within which the researcher makes sense of what he or she sees. The relationship between the experience and the story told about it is consequential to the researcher's background experience, his or her current interest, and his or her cultural perspective. It also depends on his or her intentions regarding the purpose of the research and in particular the chosen style of presenting the results. Research reports often suppress the autobiographical dimension in the process of generalising findings yet this may be the very aspect of most value to the practising teacher reading the research. In offering a piece of writing consequent to some piece of classroom based research a gesture is being made, similar to that being made by the Open University in offering a video of a lesson. That is, the reader studies the account and looks for things that resonate with his or her own experience. The issue here is not so much one of distributing 'new ideas' but rather in sharing perspectives we may gain a new way of describing learning situations and our relation to them which suggest to us new ways of working in them.

\section{REFERENCES}

Beeby, N, Burkhardt, H and Fraser, R. (1979), 'Systematic Classroom Analysis Notation', Shell Centre for Mathematics Education, University of Nottingham.

Brown, T. (1990), 'Looking at a Videotape', Mathematics Teaching, June.

Brown, T. (1991), 'Hermeneutics and Mathematical Activity', Educational Studies in Mathematics, 22, 475-480. 
Brown, A. (1987), 'Language Interaction Patterns in Lessons featuring Mathematical Investigations', unpublished PhD dissertation, University of Southampton.

Pool, P. (1990), 'A Personal Exploration into Mathematics Education in Sierra Leone', unpublished M.Phil dissertation, University of Southampton.

Ricoeur, P. (1981), Hermeneutics and the Human Sciences, Cambridge University Press, chap.8.

Walkerdine, V. (1984), 'Developmental Psychology and the Child Centred Pedagogy', in Henriques et al. Changing the Subject, London: Methuen.

Manchester Metropolitan University

Didsbury School of Education

799 Wilmslow Road

Didsbury, Manchester, M20 8RR 